\title{
Avaliação radiográfica do comportamento dos terceiros molares inferiores em pacientes tratados ortodonticamente com e sem extrações de primeiros pré-molares*
}

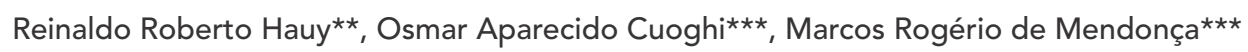

\begin{abstract}
Resumo
Objetivo: o presente trabalho objetivou avaliar as alterações ocorridas nas posições dos terceiros molares inferiores em pacientes tratados ortodonticamente com extrações dos primeiros pré-molares. Metodologia: utilizou-se 80 ortopantomografias, obtidas no pré e pós-tratamento ortodôntico corretivo de 40 pacientes, divididos em dois grupos. O grupo 1 constou de 20 pacientes (13 femininos e 7 masculinos) que se submeteram a tratamento ortodôntico com extrações de primeiros pré-molares. O grupo 2 foi constituído por 20 pacientes ( 13 femininos e 7 masculinos) tratados ortodonticamente sem extrações. Foram estabelecidas medidas angulares, com o objetivo de avaliar as inclinações e medidas lineares, para analisar as modificações no sentido vertical dos terceiros molares. Todos os dados foram mensurados duas vezes, cujos valores médios foram submetidos dos testes $t$ emparelhado e teste $t$ independente. Resultados: os resultados mostraram diferenças estatisticamente significantes $(p<0,01)$ entre as medidas angulares finais e iniciais do grupo 1 . Conclusões: concluiu-se que os deslocamentos verticais dos terceiros molares inferiores são semelhantes nos casos tratados ortodonticamente com e sem extrações e que estes elementos dentários tornam-se mais verticalizados ao final da terapia realizada com extrações de primeiros pré-molares inferiores.
\end{abstract}

Palavras-chave: Movimentação dentária. Terceiro molar. Extrações dentárias.

\section{INTRODUÇÃO E REVISÃO DE LITERATURA}

A posição dos terceiros molares gera muitas controvérsias no contexto clínico-científico, no que diz respeito ao procedimento mais adequado a ser adotado diante da sua presençç ${ }^{3,5,24}$. Um dilema muito grande que aflige os ortodontistas con- tinua sendo a decisão quanto à realização ou não das extrações dos terceiros molares, tanto para fins profiláticos como para evitar interferências durante ou após a terapia estabelecida ${ }^{3,14,28}$.

De acordo com Schulhof ${ }^{26}$, a presença do terceiro molar inferior deve ser abordada de diferen-

Trabalho de Dissertação (Mestrado) apresentado ao Programa de Pós-graduação em Odontologia, Área de concentração - Ortodontia, da Faculdade de Odontologia de Araçatuba - UNESP.

** Mestre em Ortodontia pela Faculdade de Odontologia de Araçatuba - UNESP.

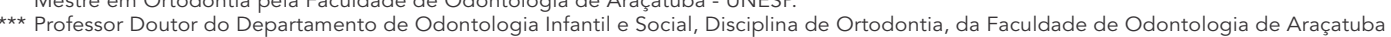
- UNESP. 
tes formas e, portanto, apresentam características individuais, tornando inviável a adoção de um protocolo único. Para muitos pacientes, este fato constitui motivo de preocupação, devido à possibilidade futura da realização de uma cirurgia, pois - segundo Bjork et al. ${ }^{4}$ - aproximadamente $45 \%$ da população apresentam terceiros molares impactados, sendo que, para muitos desses pacientes, a extração proporciona grandes desconfortos e pode ocasionar fratura mandibular ou injúrias aos segundos molares ${ }^{3}$.

O momento ideal para a realização da extração dos terceiros molares, freqüentemente, gera muitas dúvidas aos profissionais ${ }^{5,25}$. A resposta para esse questionamento dependerá da análise de muitos fatores que influenciam na tomada desta decisão. Em muitos pacientes, por exemplo, os segundos molares ainda não irromperam no arco dentário e os terceiros molares apresentam uma quantidade limitada de calcificação, dificultando a realização de um prognóstico mais consistente.

A presença dos terceiros molares pode apresentar aspectos positivos e negativos. Dentre os aspectos positivos, pode ser citada a possibilidade desse elemento dentário ser utilizado na substituição de um primeiro ou segundo molar extraído, o fornecimento de suporte para a articulação temporomandibular (ATM) ou, ainda, pode ser utilizado como auxílio para as próteses dentárias, haja vista que a ocorrência de alguns problemas associados à ATM têm sido atribuídos ao mal posicionamento dos terceiros molares inferiores $23,25,27,29$.

$\mathrm{Na}$ avaliação dos fatores negativos, a presença dos terceiros molares pode ser relacionada ao acúmulo de detritos alimentares, contribuindo, assim, para o estabelecimento de processos patológicos, como cáries, pericoronarites, outras doenças periodontais e até a formação de cistos. Ainda existe a possibilidade de diferenciação do epitélio remanescente para carcinoma espinocelular, devido à sua impactação ${ }^{12,15,27}$.

Os fatores negativos da presença dos terceiros molares no arco dentário facilmente sobrepujam os positivos, resultando, freqüentemente, nas suas extrações em, aproximadamente, $75 \%$ da população que procura atendimento odontológico ${ }^{26}$.

O relacionamento da erupção do terceiro molar inferior com a presença do apinhamento ânteroinferior tem sido abordado com muita freqüência na literatura ${ }^{1,3,4,13,15,20,24}$. Existe uma variação muito grande de resultados, não existindo um consenso no que se refere à influência dos terceiros molares no posicionamento dos incisivos inferiores. Muitos pacientes reportam que a primeira observação do apinhamento ântero-inferior coincide com a erupção dos terceiros molares inferiores e procuram o profissional por este motivo.

A previsão de impactação dos terceiros molares também gera controvérsias na literatura científica, devido ao fato de que nenhuma ocorrência biológica pode ser pré-estabelecida com absoluta certeza $^{9,12,14,19,22,24,25}$.

Diante do exposto, torna-se coerente afirmar que os terceiros molares inferiores podem se comportar de várias formas, de acordo com o seu posicionamento e o espaço disponível ${ }^{24,26}$. Conseqüentemente, a avaliação de vários fatores determinará um prognóstico mais correto para os diversos casos, bem como uma maior segurança nos procedimentos terapêuticos a serem adotados, tanto para os profissionais das várias especialidades como para os pacientes.

Desta forma, o objetivo deste trabalho foi avaliar radiograficamente o comportamento angular no sentido mesiodistal e vertical dos terceiros molares inferiores, em casos tratados ortodonticamente com e sem extrações de primeiros prémolares.

\section{MATERIAL E MÉTODOS \\ Material}

Inicialmente, foram analisadas 130 documentações ortodônticas e 80 radiografias ortopantomográficas de 40 pacientes, de ambos os gêneros, tratados ortodonticamente, selecionadas aleatoriamente na Disciplina de Ortodontia da Facul- 
dade de Odontologia de Araçatuba - UNESP. As radiografias foram obtidas em um mesmo aparelho (Rotograph Plus). Como critério de seleção das radiografias iniciais, foi estabelecido que os terceiros molares inferiores deveriam apresentar o desenvolvimento total de suas coroas, correspondente ao estágio 6 da classificação de Nolla ${ }^{18}$. Além disso, os primeiros molares inferiores permanentes deveriam apresentar as suas raízes e coroas completamente formadas ou seja, correspondente ao estágio 10 da classificação de Nolla ${ }^{18}$. Posteriormente, as radiografias foram divididas em 2 grupos, da seguinte forma:

- Grupo 1: 40 radiografias ortopantomográficas de 20 pacientes que realizaram o tratamento ortodôntico com extrações de primeiros pré-molares, sendo 20 radiografias correspondentes ao início e 20 ao final do tratamento. Neste grupo, 7 pacientes eram do gênero masculino e 13 do feminino. A faixa etária no início do tratamento variou de 11 anos e 3 meses a 18 anos e 3 meses de idade, com média de 13 anos e 10 meses. Ao final do tratamento, a faixa etária variou de 14 anos e 11 meses a 22 anos e 9 meses de idade, com média de 18 anos e 1 mês.

- Grupo 2: 40 radiografias ortopantomográficas de 20 pacientes que realizaram o tratamento ortodôntico sem extrações dentárias, sendo 20 radiografias correspondentes ao início e 20 ao final do tratamento. Neste grupo, 7 pacientes eram do gênero masculino e 13 do gênero feminino. A faixa etária no início do tratamento variou de 9 anos e 7 meses a 18 anos e 3 meses de idade, com média de 13 anos e 11 meses. Ao final do tratamento, a faixa etária variou de 12 anos e 1 mês a 23 anos e 11 meses de idade, com média de 16 anos e 8 meses.

\section{Métodos}

O posicionamento dos terceiros molares no início e final do tratamento ortodôntico foi avaliado por meio de medidas angulares e lineares (Fig. $1,2)$ objetivando estabelecer as possiveis alterações relacionadas com a sua inclinação mesiodistal e seu posicionamento no sentido vertical. Para a realização das mensurações angulares e lineares, inicialmente, adaptou-se sobre as radiografias folhas de papel acetato semi-transparente, sobre as quais foram delimitadas estruturas anatômicas, de acordo com o preconizado por Tavano et al. ${ }^{30}$

A partir do traçado anatômico do côndilo, ramo, corpo mandibular, terceiros molares e forame mentoniano de ambos os lados, foram determinadas as seguintes linhas de referência (Fig. 1):

- A: linha unindo os pontos centrais dos contornos dos forames mentonianos, denominada linha intermentoniana;

- B: linha formada pela união das cúspides mesial e distal dos terceiros molares inferiores, ou seja, o plano oclusal do terceiro molar inferior;

- C: linha do ponto central da coroa dos terceiros molares inferiores perpendicular à linha intermentoniana.

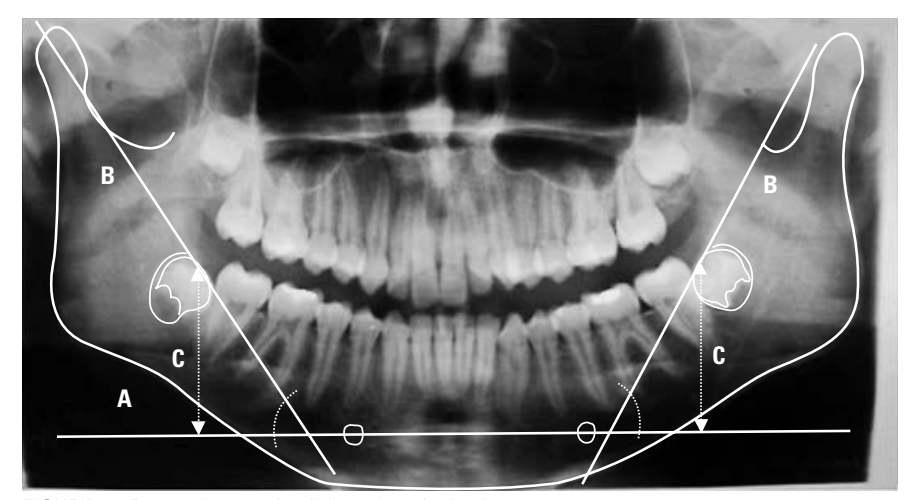

FIGURA 1 - Determinação das linhas de referência. 
As medidas angulares foram obtidas a partir da mensuração do ângulo formado pela intersecção da linha intermentoniana com o plano oclusal dos terceiros molares inferiores (Fig. 2), para analisar as inclinações dos terceiros molares inferiores. Os valores das medidas angulares foram denominados da seguinte forma:

- Maic: medida angular inicial do grupo com extrações;

- Mafc: medida angular final do grupo com extrações;

- Mais: medida angular inicial do grupo sem extrações;

- Mafs: medida angular final do grupo sem extrações.
As medidas lineares foram mensuradas por meio da distância perpendicular do ponto central da coroa dos terceiros molares à linha intermentoniana (Fig. 3). Estas medidas analisam as alterações no sentido vertical dos terceiros molares inferiores e foram denominadas como:

- Mlic: medida linear inicial do grupo com extrações;

- Mlfc: medida linear final do grupo com extrações;

- Mlis: medida linear inicial do grupo sem extrações;

- Mlfs: medida linear final do grupo sem extrações.

Com o objetivo de eliminar as possíveis dis-

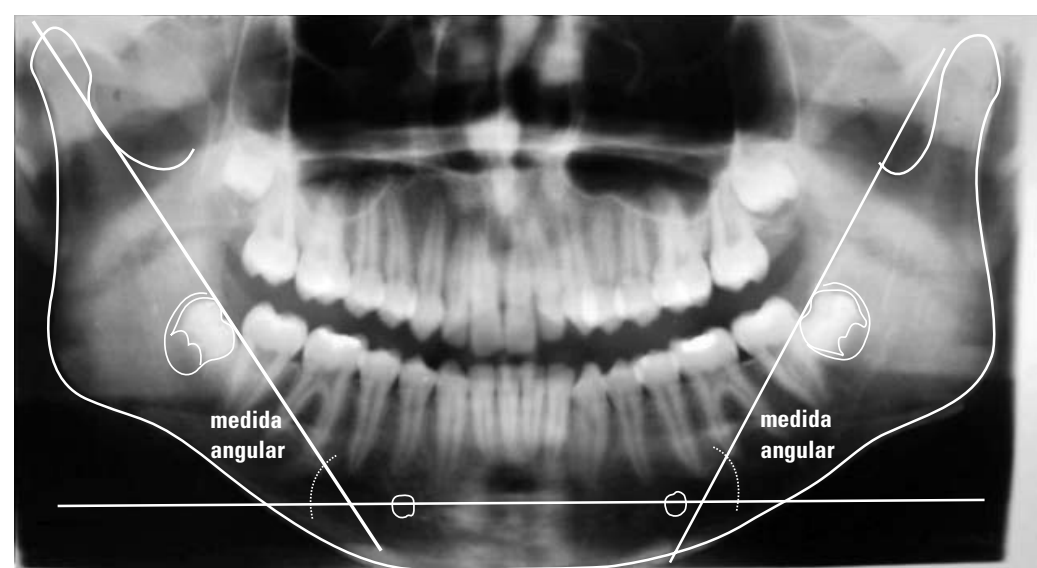

FIGURA 2 - Estabelecimento das medidas angulares.

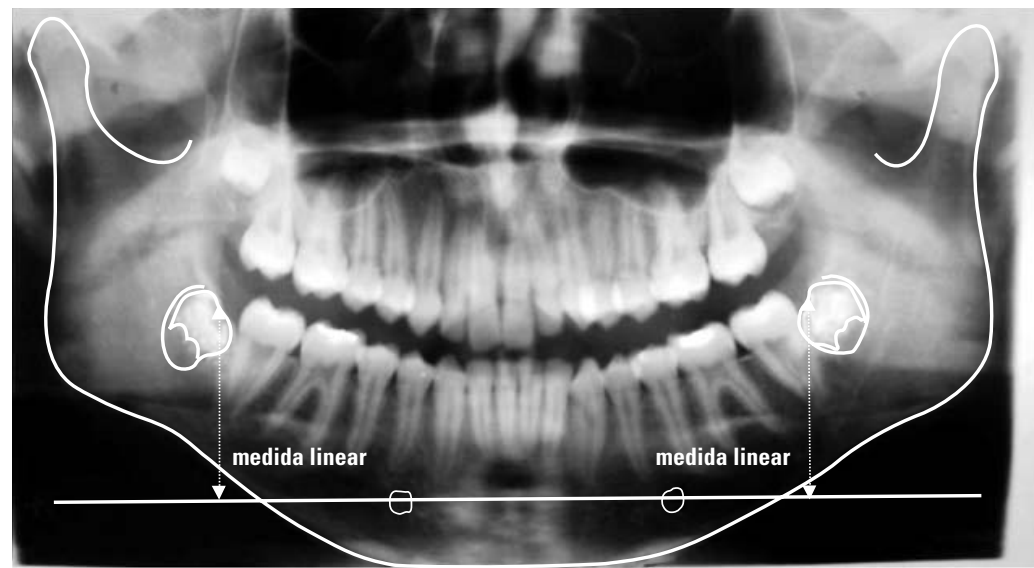

FIGURA 3 - Estabelecimento das medidas lineares. 
torções, que podem ocorrer quando avaliadas as medidas lineares obtidas de radiografias panorâmicas, utilizou-se como critério a mensuração da distância entre a ponta da cúspide mesial do primeiro molar permanente inferior até o ápice da raiz mesial deste mesmo elemento dentário (Fig. 4). Esta distância foi mensurada nas radiografias obtidas no início e no final do tratamento. Os valores obtidos foram, então, submetidos à análise estatística, na qual foi evidenciada diferença estatisticamente significante entre o comprimento do primeiro molar inferior permanente nas radiografias iniciais e finais, confirmando, desta forma, a influência da distorção da imagem radiográfica para as medidas lineares (Tab. 1, 2, 3). A partir destes resultados, foi necessário obter-se uma metodologia para eliminar tais distorções. Como critério, calculou-se a porcentagem da distorção entre as medidas de todas as radiografias panorâmicas. $\mathrm{O}$ índice porcentual de distorção obtido foi, então, aplicado sobre as medidas lineares finais individuais de ambos os grupos, eliminando, desta forma, as interferências da distorção da imagem radiográfica.

Após a aplicação do índice de distorção para as medidas lineares, realizou-se a análise estatística

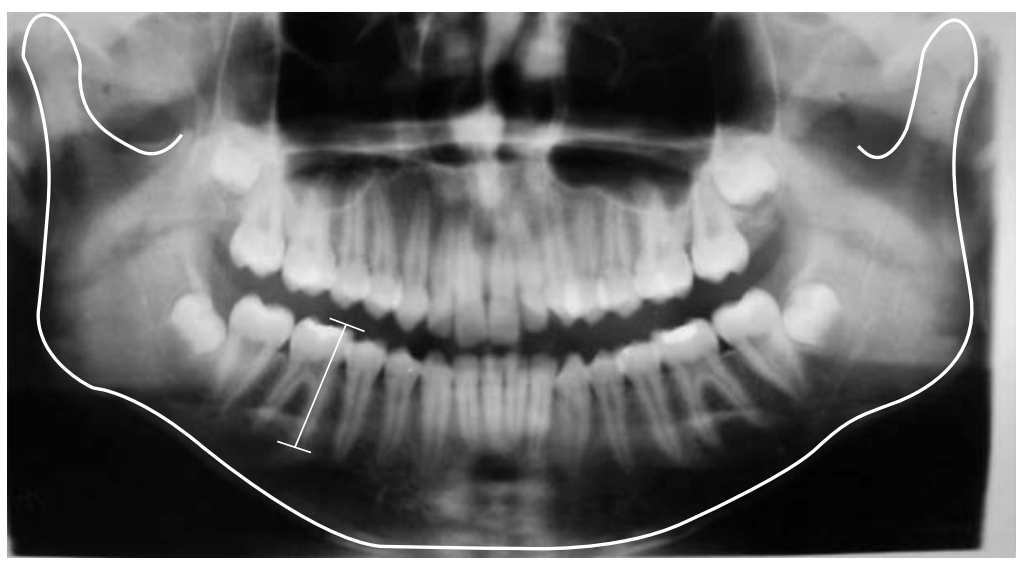

FIGURA 4 - Distância da ponta da cúspide mesial ao ápice da raiz mesial do primeiro molar inferior.

Tabela 1 - Valores médios iniciais, finais e os desviospadrão da distância entre a ponta da cúspide mesial até 0 ápice da raiz mesial do primeiro molar inferior permanente do grupo 1.

\begin{tabular}{ccc}
\hline grupo 1 & valor médio inicial & valor médio final \\
\hline média $(\mathrm{mm})$ & 30,65 & 28,22 \\
d.p. & 2,920 & 2,588 \\
\hline
\end{tabular}

Total: 20 radiografias iniciais e 20 radiografias finais.
Tabela 2 - Valores médios iniciais, finais e os desviospadrão da distância entre a ponta da cúspide mesial até 0 ápice da raiz mesial do primeiro molar inferior permanente do grupo 2.

\begin{tabular}{ccc}
\hline grupo 2 & valor médio inicial & valor médio final \\
\hline média $(\mathrm{mm})$ & 29,07 & 27,15 \\
d.p. & 1,779 & 2,027 \\
\hline
\end{tabular}

Total: 20 radiografias iniciais e 20 radiografias finais.

Tabela 3 - Valores de t, p e a decisão estatística resultante das comparações entre os valores médios da distância entre a ponta da cúspide mesial até o ápice da raiz mesial do primeiro molar inferior permanente obtidos das radiografias iniciais e finais de ambos os grupos.

\begin{tabular}{cccc}
\hline grupos & $\mathbf{t}$ & $\mathbf{p}$ & decisão estatística \\
\hline grupo 1 & 5,035 & $\mathrm{p}<0,0001$ & significante $^{* *}$ \\
grupo 2 & 3,536 & $\mathrm{p}=0,0022$ & significante* $^{*}$ \\
\hline
\end{tabular}

* Significante ao nível de $1 \%$.

** Significante ao nível de $0,01 \%$. 
para a obtenção do nível de análise de significância, onde foram comparados os valores obtidos em cada grupo, bem como entre os grupos.

\section{RESULTADOS}

Após a obtenção dos valores das medidas angulares e lineares do início e final de tratamento ortodôntico realizado com e sem extrações, aplicou-se o tratamento estatístico para a obtenção dos valores médios e seus respectivos níveis de significância.

As tabelas 4 e 5 mostram as médias, desviospadrão, valores máximo e mínimo das medidas lineares dos terceiros molares inferiores, no início e ao final dos tratamentos ortodônticos realizados com e sem extrações dentárias. A tabela 4 mostra os resultados encontrados no grupo 1, composto pelos casos tratados com extrações dentárias, apresentando, como média para as medidas angulares do início de tratamento (Maic), o valor de 52,02 graus, com desvio-padrão de 11,36 , enquanto para as medidas angulares do final do tratamento (Mafc) a média foi de 41,56 graus, com desvio-padrão de 13,66 . As médias encontradas para as medidas lineares iniciais do grupo com extrações (Mlic) e medidas lineares finais com extrações (Mlfc) fo- ram respectivamente $37,65 \mathrm{~mm}$ (desvio-padrão de 5,49 ) e $38,95 \mathrm{~mm}$ (desvio-padrão de 5,18 ).

Por outro lado, estão representados na tabela 5 os valores médios das medidas angulares e lineares do grupo 2, composto por radiografias de pacientes que foram tratados ortodonticamente sem extrações. A medida angular inicial deste grupo (Mais) apresentou um valor médio de 47,73 graus, com desvio-padrão de 11,38. Da mesma forma, esta tabela apresenta ainda os valores médios das medidas angulares finais do grupo 2 (Mafs), que revelaram um valor médio de 45,99 graus, com desvio-padrão de 15,61. A observação destas tabelas ainda nos mostra que o grupo 2 apresentou para as medidas lineares iniciais (Mlis) um valor médio de $36,85 \mathrm{~mm}$ com desvio-padrão de 3,86 . Entretanto, as medidas lineares e finais deste grupo apresentaram-se com valor médio de $37,33 \mathrm{~mm}$ e com desvio-padrão de 6,60.

Posteriormente à obtenção dos valores médios, realizou-se o teste $\mathrm{t}$ emparelhado para observar o nível de significância nos grupos com e sem extrações (Grupos 1 e 2).

Os resultados estatísticos das comparações realizadas dentro de cada grupo estão demonstrados na tabela 6 . Nesta análise foram detectadas dife-

Tabela 4 - Valores angulares e lineares médios, desvios-padrão, diferenças entre inicial e final e valores máximos e mínimos do grupo com extrações.

\begin{tabular}{ccccccc}
\hline dados & MAIC (graus) & MAFC (graus) & diferença & MLIC (mm) & MLFC (mm) & diferença \\
\hline média & 52,02 & 41,56 & 10,48 & 37,65 & 38,95 & $-1,66$ \\
d.p. & 11,36 & 13,66 & 12,44 & 5,49 & 5,18 & 5,48 \\
mínimo & 38,00 & 14,00 & $-37,00$ & 23,00 & 28,5 & $-13,50$ \\
máximo & 81,00 & 77,00 & 29,50 & 53,00 & 52,5 & 8,00 \\
\hline
\end{tabular}

Total: 20 radiografias iniciais e 20 radiografias finais.

Tabela 5 - Valores angulares e lineares médios, desvios-padrão, diferenças entre inicial e final e valores máximos e mínimos do grupo sem extrações.

\begin{tabular}{ccccccc}
\hline dados & MAIS (graus) & MAFS (graus) & diferença & MLIS (mm) & MLFS (mm) & diferença \\
\hline média & 47,73 & 45,99 & 1,74 & 36,85 & 37,33 & $-0,48$ \\
d.p. & 11,38 & 15,61 & 11,26 & 3,86 & 6,60 & 6,19 \\
mínimo & 28,00 & 16,00 & $-26,00$ & 29,00 & 21,00 & $-13,50$ \\
máximo & 91,00 & 89,00 & 27,00 & 51,00 & 51,00 & 14,50 \\
\hline
\end{tabular}

Total: 20 radiografias iniciais e 20 radiografias finais. 
renças estatisticamente significantes em nível de $1 \%$, quando comparados os valores das medidas angulares iniciais com extrações (Maic) com os valores das medidas angulares do final de tratamento (Mafc).

Para avaliar o nível de significância das medidas lineares e angulares entre os grupos 1 e 2 , aplicouse o teste $t$ independente (Tab. 7).

Após a obtenção dos resultados, não foram de-

Tabela 6 - Valores t, p e decisão estatística resultante das comparações entre os valores iniciais e finais de cada grupo por meio do teste t emparelhado.

\begin{tabular}{cccc}
\hline grupos & $\mathbf{t}$ & $\mathbf{p}$ & decisão estatística \\
\hline Maic x Mafc & 5,326 & $\mathrm{p}<0,0001$ & significante* \\
Mlic x Mlfc & 1,920 & $\mathrm{p}=0,0622$ & n.s. \\
Mais x Mafs & 0,976 & $\mathrm{p}=0,3353$ & n.s. \\
Mlis x Mlfs & 0,4851 & $\mathrm{p}=0,6303$ & n.s. \\
\hline
\end{tabular}

n.s. = não significante

* significante ao nível de 0,01\%.

Tabela 7 - Valores t, p e decisão estatística resultante das comparações entre os valores angulares, lineares iniciais e finais dos grupos com e sem extração, por meio do teste $t$ independente.

\begin{tabular}{cccc}
\hline grupos & $\mathbf{t}$ & $\mathbf{p}$ & decisão estatística \\
\hline Maic x Mais & 1,696 & $\mathrm{p}=0,0938$ & n.s. \\
Mlic x Mlis & 0,4118 & $\mathrm{p}=0,6816$ & n.s. \\
Mafc x Mafs & 1,349 & $\mathrm{p}=0,1812$ & n.s. \\
Mlfc x Mlfs & 1,225 & $\mathrm{p}=0,2244$ & n.s. \\
\hline n.s. = não significante. & & &
\end{tabular}

Tabela 8 - Resultado do erro do método calculado segundo a fórmula preconizada por Dahlberg.

\begin{tabular}{cc}
\hline grandeza & Dahlberg \\
\hline Maic & 0,38 \\
Mais & 0,29 \\
Mafc & 0,29 \\
Mafs & 0,44 \\
Mlic & 0,40 \\
Mlis & 0,41 \\
Mlfc & 0,29 \\
Mlfs & 0,27 \\
\hline
\end{tabular}

tectadas diferenças estatisticamente significantes, quando comparados os valores das medidas angulares iniciais e finais entre ambos os grupos, bem como quando comparados os valores das medidas lineares iniciais e finais entre os 2 grupos.

Para a verificação da confiabilidade e da reprodutibilidade das mensurações, calculou-se o erro do método, de acordo com a seguinte fórmula matemática preconizada por Dahlberg: $\mathrm{E}^{2}=\sum \mathrm{d}^{2} / 2 \mathrm{n}$, onde " $E$ " indica o erro a ser calculado, "d" indica a diferença entre as duas mensurações para a mesma grandeza e " $n$ " indica o número de elementos mensurados. Os resultados obtidos encontram-se expressos na tabela 8 .

Pelo método de Dalberg, observou-se que os erros das grandezas foram pequenos, com valores menores do que o valor da escala utilizada para a mensuração, isto é, $0,5^{\circ}$ para as grandezas angulares e $0,5 \mathrm{~mm}$ para as grandezas lineares.

\section{DISCUSSÃO}

O posicionamento e, conseqüentemente, as movimentações fisiológicas dos terceiros molares estão intimamente relacionados com a disponibilidade de espaço no arco dentário. Desta forma, os terceiros molares inferiores têm se apresentado como um dos dentes que acarretam maiores problemas, tanto para os pacientes como para os profissionais. A obtenção de espaço para estes dentes depende do crescimento mandibular com reabsorção na borda anterior do ramo, da tendência de mesialização dos dentes posteriores, devido às alterações relacionadas com a oclusão, e dos procedimentos terapêuticos realizados com extrações dentárias $8,9,16,21,22,25,27$.

Dentre os diversos procedimentos terapêuticos ortodônticos, em algumas situações, pode-se obter espaço para os terceiros molares inferiores por meio das extrações dentárias ou pela movimentação dos dentes anteriores aos terceiros molares ${ }^{7,16,18,22}$.

Apesar da existência de vários trabalhos apresentados na literatura a respeito dos deslocamen- 
tos dos terceiros molares inferiores ${ }^{9,18,23}$, este estudo objetivou verificar o comportamento destes dentes nas situações que envolvem os tratamentos ortodônticos que são realizados com extrações de pré-molares e comparar com os tratamentos que visam a correção da má oclusão, nos quais não havia necessidade da realização de nenhuma extração nos arcos dentários.

Como metodologia, optou-se pela avaliação em radiografias ortopantomográficas ou panorâmicas, haja vista que este método auxiliar de diagnóstico, utilizado na prática ortodôntica, faz parte da documentação freqüentemente solicitada aos pacientes para o início do tratamento. Além disso, observa-se que este método radiográfico é de boa aceitação pelos pacientes, sendo relevante no sentido de evidenciar os estágios de desenvolvimento e erupção dentáriaa ${ }^{11,30,31}$.

Ainda sobre a importância da radiografia panorâmica, Capelloza Filho et al. ${ }^{6}$ enfatizaram a importância deste método para observar o posicionamento mesiodistal dos dentes, tanto no início como ao final do tratamento ortodôntico.

O presente estudo objetivou avaliar o comportamento da movimentação dos terceiros molares inferiores, observando as suas mudanças de inclinação, ou seja, as alterações no sentido mesiodistal, coadjuvante à analise do seu potencial de deslocamento vertical.

Para avaliar a inclinação destes elementos dentários, optou-se por mensurar o ângulo formado pela intersecção da linha que une os dois forames mentonianos (linha intermentoniana) com a linha que tangencia as cúspides dos terceiros molares inferiores direito e esquerdo, estabelecido como o plano oclusal dos terceiros molares inferiores (Fig. 3). Este critério está embasado na literatura, que relata que as medidas angulares avaliadas em radiografias panorâmicas podem ser utilizadas com muita segurança na prática clínica ${ }^{6,11,30,31}$.

Para avaliar o comportamento vertical dos terceiros molares inferiores, ou seja, os possíveis movimentos relacionados com a erupção dentária, este trabalho utilizou a distância linear da porção média da coroa dos terceiros molares inferiores até a linha intermentoniana.

A análise estatística, realizada comparando-se as medidas angulares iniciais entre os grupos tratados com e sem extrações, demonstra uma homogeneidade entre os valores obtidos, determinando, desta forma, uma similaridade entre as medidas angulares iniciais de ambos os grupos (Tab. 7). Portanto, as diferenças ocorridas ao final do tratamento devem-se exclusivamente aos efeitos proporcionados pela terapia ortodôntica utilizada nos casos avaliados neste trabalho, para ambos os grupos.

A referência dos forames mentonianos e, conseqüentemente, o traçado da linha intermentoniana foram comprovados por Tavano et al ${ }^{30}$ como sendo um método confiável, uma vez que são estruturas fáceis de serem visualizadas radiograficamente e não são influenciadas pelo crescimento, bem como pelas terapias ortodônticas.

$\mathrm{Na}$ literatura, existem algumas controvérsias sobre o nível de distorção das radiografias ortopantomográficas para a realização de mensurações lineares ${ }^{2,31}$. Objetivando eliminar a ocorrência dessas distorções, selecionou-se a dimensão dentária estabelecida pela distância coroa-ápice dos primeiros molares inferiores (Fig. 4). Optou-se pela avaliação destes elementos dentários por serem os primeiros dentes permanentes a irromperem, por estarem completamente desenvolvidos na fase inicial de tratamento da amostra estudada e por não apresentarem influência do crescimento, no que diz respeito à sua medida final, ou seja, a distância coroa-ápice, o que poderia ocorrer se fosse selecionada alguma região de estrutura óssea. Após a obtenção dos valores das dimensões destes dentes, nas radiografias do início e final do tratamento ortodôntico do mesmo paciente, realizou-se a análise estatística. $\mathrm{O}$ resultado confirmou a presença de distorções significantes nos tamanhos dessas estruturas dentárias, de acordo com o observado na literatura ${ }^{2,31}$. 
A partir dessa avaliação, foi estabelecida, para cada radiografia, a porcentagem de distorção ocorrida. Os valores porcentuais foram então aplicados para as medidas lineares finais obtidas diretamente das radiografias e, desta forma, foram eliminadas as interferências das distorções da imagem radiográfica sobre os resultados finais. Conseqüentemente, pode-se afirmar que as medidas lineares das radiografias do final de tratamento, utilizadas para realizar a análise estatística, não foram as reais, mas sim com as modificações determinadas pela porcentagem de distorções ocorridas.

Para facilitar a elucidação e o entendimento dos resultados obtidos, serão discutidas separadamente as medidas angulares e lineares.

\section{Medidas angulares}

$\mathrm{O}$ valor médio encontrado para os casos tratados com extrações de pré-molares (Grupo 1) no início do tratamento foi de $52^{\circ}$, enquanto ao final do tratamento o valor médio foi de $41,5^{\circ}$ (Gráf. 1). Esta alteração foi estatisticamente significante, demonstrando uma tendência para a redução do ângulo avaliado, estabelecendo uma alteração acentuadamente favorável no sentido do posicionamento mesiodistal dos terceiros molares inferiores. Este resultado demonstra a ocorrência de um giro ou deslocamento considerável para distal dos terceiros molares inferiores, adquirindo um posicionamento mais verticalizado.

Para os casos tratados sem extrações de prémolares, o valor médio para a medida angular no início foi de $47,7^{\circ}$ e, portanto, maior do que aquele encontrado ao final do tratamento, que foi de 45,9 $9^{\circ}$ (Gráf. 1). Entretanto, este nível de alteração não foi considerado estatisticamente significante, quando submetido à análise estatística. Mesmo sem significância estatística, estes valores sugerem também uma tendência para a verticalização dos terceiros molares inferiores nos casos tratados com e sem extrações. As modificações provenientes da verticalização dos terceiros molares inferiores estão provavelmente relacionadas com o cresci- mento e desenvolvimento craniofacial associado à reabsorção da borda anterior do ramo da mandíbula ${ }^{9,16}$. Além disso, Richardson ${ }^{19}$ mencionou que a angulação pode ser influenciada pela diferença do potencial de desenvolvimento que pode ocorrer entre as raízes mesial e distal dos terceiros molares.

De acordo com os valores encontrados, observou-se que a quantidade de alteração angular entre as medidas iniciais e finais foi acentuadamente maior no grupo tratado com extrações dentárias (25\%), quando comparado com o grupo tratado sem extrações dentárias (4\%), ou seja, aproximadamente 6 vezes maior, indicando a tendência para uma maior verticalização dos terceiros molares inferiores após o tratamento realizado com extrações de primeiros pré-molares (Gráf. 1).

No presente trabalho, ficou caracterizado que - independentemente de outros fatores que possam favorecer a modificação angular dos terceiros molares inferiores, como o crescimento, a reabsorção na borda anterior do ramo mandibular e a tendência de mesialização dos dentes posteriores - a terapia ortodôntica realizada com extrações de pré-molares proporciona um ambiente mais favorável para que ocorra uma verticalização de

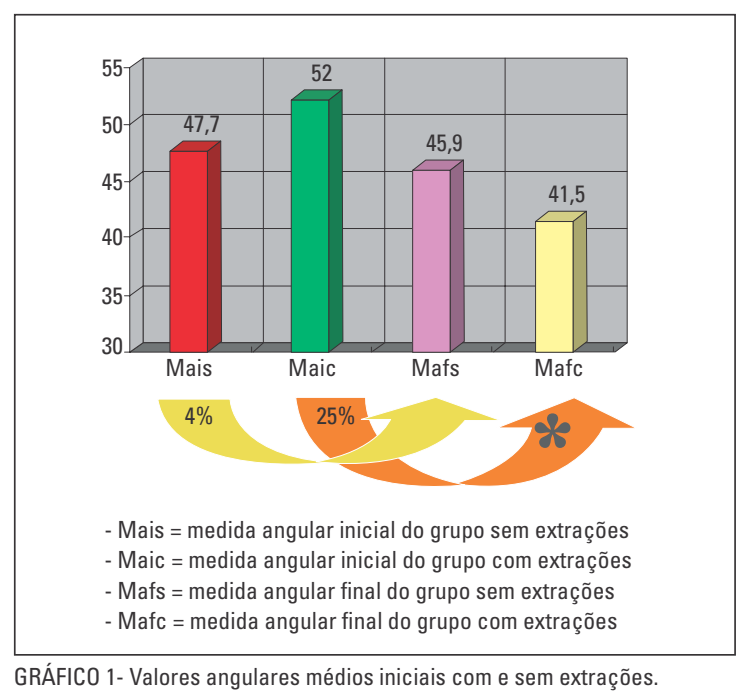

* Estatisticamente significante ao nível de 0,01\%. 
forma significante desses elementos dentários. Da mesma forma, Silling ${ }^{27}$ considerou que a perda de dentes anteriores aos terceiros molares diminui a incidência de sua impactação, permitindo uma rotação normal de sua coroa até o posicionamento adequado para a sua erupção.

\section{Medidas lineares}

Como foi mencionado anteriormente, a obtenção das medidas lineares visa evidenciar as modificações dos terceiros molares inferiores no sentido vertical, mais especificamente o potencial de deslocamento eruptivo.

Diante dos resultados obtidos, observou-se que os valores médios no final do tratamento ortodôntico realizado sem extrações $(37,3 \mathrm{~mm})$ foram maiores do que no início do tratamento $(36,8 \mathrm{~mm})$. Da mesma forma, no grupo tratado com extrações dentárias, o valor médio no final do tratamento foi de $38,9 \mathrm{~mm}$ e no início foi de $37,6 \mathrm{~mm}$ (Gráf. 2). Apesar da comparação estatística entre o início e final de tratamento nos 2 grupos não apresentar diferenças estatisticamente significantes, ficou caracterizada uma tendência para um deslocamento dos terceiros molares inferiores no sentido vertical, ou seja, no sentido extrusivo, tanto nos casos que envolveram extrações dentárias como na-

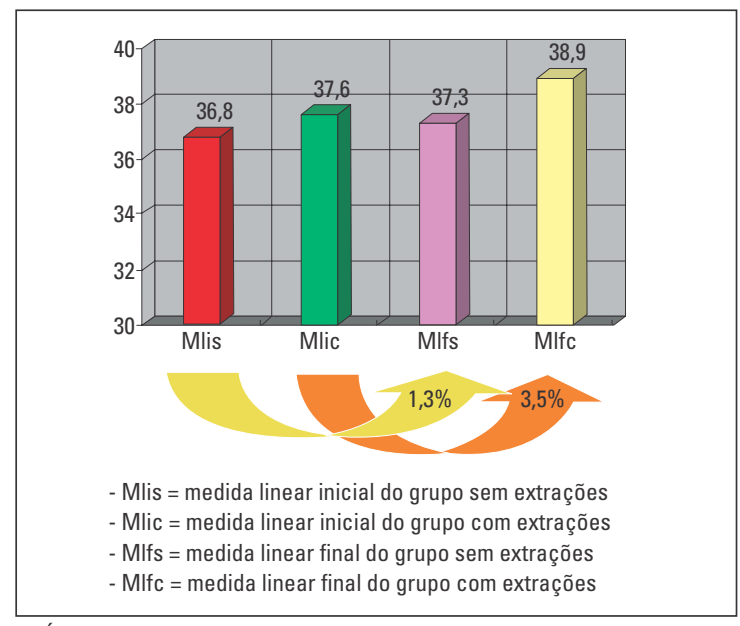

GRÁFICO 2 - Valores lineares médios iniciais com e sem extrações. queles em que a terapia ortodôntica é realizada sem extrações. Entretanto, convém salientar que as modificações lineares foram maiores nos casos tratados com extrações (Grupo 2), pois as medidas lineares finais do grupo sem extrações se alteraram apenas 1,3\% em relação ao seus valores iniciais, enquanto no grupo tratado com extrações essa alteração foi de 3,5\% (Gráf. 2), ou seja, quase 3 vezes mais, sugerindo uma pequena tendência para maior movimento no sentido vertical dos terceiros molares inferiores nos casos tratados com extrações dentárias.

Haavikko et al. ${ }^{13}$ demonstraram, também, que as extrações de pré-molares podem estabelecer apenas uma suave alteração relacionada com a erupção dos terceiros molares, ou seja, não ocorrem deslocamentos significantes no sentido vertical. Entretanto, vários trabalhos relataram que as extrações dentárias favorecem o processo de erupção dos terceiros molares $8,16,18,22,29$.

As alterações verticais relacionadas com as medidas angulares obtidas permitem mencionar que os deslocamentos verticais não apresentam modificações expressivas, quando comparados os valores médios do início e final de tratamento ortodôntico com e sem extrações dentárias.

Durante a avaliação das medidas angulares, notou-se uma melhora significante no posicionamento dos terceiros molares, sugerindo um deslocamento no sentido distal, nos casos tratados com extrações de pré-molares, corroborando com os resultados obtidos por Meral et al. ${ }^{17}$

Associando os resultados médios lineares e angulares, pode-se estabelecer que os terceiros molares inferiores tornam-se significantemente mais verticalizados com as extrações de pré-molares, porém, este fato não interfere no deslocamento vertical, sugerindo pequeno desenvolvimento eruptivo.

Os resultados deste trabalho corroboram com aqueles obtidos por Richardson ${ }^{19}$, que demonstrou uma verticalização ou deslocamento distal dos terceiros molares em $46 \%$ dos casos contra 13\% que 
não apresentaram alteração em sua angulação e 41\% que tenderam para uma movimentação mesial.

Apesar deste estudo não ter avaliado o índice de ancoragem, o trabalho de Staggers et al..$^{29}$ mencionou que as extrações de pré-molares não influenciam o posicionamento dos terceiros molares, mas talvez a mecânica e, conseqüentemente, o tipo de ancoragem utilizada possam levar a alterações no posicionamento destes elementos dentários.

Desta forma, sugere-se que as exodontias dos terceiros molares inferiores possam ser proteladas para o final do tratamento ortodôntico, principalmente nos casos onde a extração de pré-molares faz parte da terapia planejada. Entretanto, outros trabalhos devem ser realizados com o intuito de avaliar as modificações angulares e lineares dos terceiros molares inferiores, observando novos dados, como o comportamento das unidades de ancoragem, padrão de crescimento, tipos de má oclusão, relacionando-os com o comportamento destes dentes em longo prazo.

Como síntese, este estudo demonstrou que: 1) os valores médios relacionados com as alterações no sentido vertical não apresentaram diferenças estatisticamente significantes entre o início e final do tratamento ortodôntico realizado com ou sem extrações; 2) os valores angulares não apresen- taram diferenças estatisticamente significantes entre o início e final de tratamento ortodôntico sem extrações de pré-molares e 3) ocorreram alterações estatisticamente significantes entre o início e o final de tratamento dos casos que se submeteram à terapia ortodôntica com extrações de pré-molares.

\section{CONCLUSÕES}

Diante da metodologia empregada, conclui-se que:

- Os deslocamentos dos terceiros molares inferiores no sentido vertical são semelhantes quando comparados os casos tratados ortodonticamente com e sem extrações de pré-molares;

- Os terceiros molares inferiores apresentam pequenos deslocamentos para distal ao final dos tratamentos ortodônticos que não envolvem extrações de pré-molares;

- Os terceiros molares inferiores movimentamse para distal de forma significante e, portanto, tornam-se mais verticalizados ao final das terapias ortodônticas realizadas com extrações de pré-molares.

\title{
Radiographic evaluation of the behavior of lower third molars in patients that were orthodontically treated with and without first premolars extractions
}

\begin{abstract}
Aim: The aim of this study is to evaluate the positional changes occurred with lower third molars in patients treated orthodontically with extractions of first premolars. Methods: A total of 80 panoramic radiographs obtained at pre and post-treatment periods from 40 patients, were divided into 2 groups: group 1 were composed by 20 patients (13 female and 7 male) that were submitted to orthodontic treatment with extraction of first premolars; group 2 were composed by 20 patients ( 13 female and 7 male) that were submitted to orthodontic treatment without extractions. Angular measurements were established to evaluate the tipping and linear measurements were established to analyze the vertical changes of the lower third molars. All data were measured twice and averaged, then submitted to paired and unpaired test. Results: Significant differences were found between the initial and final angular measurements of group $1(p<0.01)$. Conclusions: It was concluded that similar vertical changes of lower third molars occurred when extraction and non-extraction cases were compared, and third lower molars became less tipped with the extractions of lower first premolars.
\end{abstract}

Key words: Tooth movement. Third molar. Tooth extractions. 


\section{REFERÊNCIAS}

1. ADES, A. G.; JOONDEPH, D. R.; LITTLE, R. M.; CHAPKO, M. K. Long-term study of the relationship of third molars changes in the mandibular dental arch. Am. J. Orthod. Dentofacial Orthop., St. Louis, v. 97, no. 4, p. 323-335, Apr. 1990.

2. ALMEIDA, S. M.; BOSCOLO, F. N.; MONTEBELO FILHO, A. Estudo das distorções da imagem radiográfica produzida em aparelhos panorâmicos que se utilizam dos princípios ortopantomográficos e elipsopantomográficos. Rev. Odontol. Univ. São Paulo, São Paulo, v. 9, n. 2, p. 91-99, abr./jun. 1995.

3. BISHARA, S. E. Third molars: a dilemma! Or is it? Am. J. Orthod. Dentofacial Orthop., St. Louis, v. 115, no. 6, p. 628-633, June 1999.

4. BJÖRK, A.; JENSEN, E.; PALLING, M. Mandible growth and third molar impaction. Acta Odontol. Scand., Oslo, v. 14, p. 231-272, Nov. 1956.

5. BRAMANTE, M. A. Controversies in Orthodontics. Dent. Clin. North Am., Chicago, v. 34, no. 1, p. 91-102, Jan. 1990.

6. CAPELOZZA FILHO, L. et al. Angulação dentária após o tratamento ortodôntico pela técnica de Andrews e Edgewise: avaliação pela ortopantomografia. Ortodontia, São Paulo, v. 27 , n. 2 , p. 60-66, maio/ago. 1994

7. ELSEY, M. J.; ROCK, W. P. Influence of orthodontic treatment on development of third molars. Br. J. Oral Maxillofac. Surg., Edinburg, v. 38, no. 4, p. 350-353, Aug. 2000.

8. FAUBION, B. H. Effect of extraction of premolars on eruption of mandibular third molars. J. Am. Dent. Assoc., Chicago, v. 76 , no. 2, p. 316-320, Feb. 1968

9. FAYAD, J. B.; LEVY, J. C.; YAZBECK, C.; CAVEZIAN, R.; CABANIS, E. C. Eruption of third molars: relationship to inclination of adjacent molars. Am. J. Orthod. Dentofacial Orthop., St. Louis, v. 125, no. 2, p. 200-202, Feb. 2004.

10. FORSBERG, C. M.; VINGREN, B.; WESSLÉN, U. Mandibular third molar eruption in relation to available space as assessed on lateral cephalograms. Swed. Dent. J., Jönköping, v. 13, no. 1/2, p. 23-31, 1989.

11. FRYKHOLM, A.; MALMGREN, O.; SAMFORS, K. A. S.; WELANDER, U. Angular measurements in orthopantomography. Dentomaxillofac. Radiol., Houndsmills, v. 6, no. 2, p. 77-81, 1977.

12. GÜNGÖRMUS, M. Pathologic status and changes in mandibular third molar position during orthodontic treatment. J. Contemp. Dent. Pract., Cincinnati, v. 3, no. 2, p. 11-22, May 2002.

13. HAAVIKKO, K.; ALTONEN, M.; MATTILA, K. Predicting angulational development and eruption of the third molar. Angle Orthod., Appleton, v. 48, no. 1, p. 39-48, Jan. 1978.

14. HARRADINE, N. W. T.; PEARSON, M. H.; TOTH, B. The effect of extraction of third molars on late lower incisor crowding: a randomizes controlled trial. Br. J. Orthod., Oxford, v. 25, no. 2, p. 117-122, May 1998

15. KAHL, B.; GERLACH, K. L.; HILGERS, R. D. A long-term, followup, radiography evaluation of asymptomatic impacted third molars in orthodontically treated patients. Int. J. Oral Maxillofac. Surg., Copenhagen, v. 23, no. 5, p. 279-285, Oct. 1994.

16. KIM, T. W.; ARTUN, J.; BEHBEHANI, F.; ARTESE, F. Prevalence of third molar impaction in orthodontic patients treated nonextraction and with extraction of 4 premolars. Am. J. Orthod Dentofacial Orthop., St. Louis, v. 123, no. 2, p. 138-145, Feb. 2003.

17. MERAL, G. D.; SAYSEL, M. Y.; KOCADERELLI, I.; TASAR, F. The effects of first premolar extractions on third molar angulations. Angle Orthod., Appleton, v. 75, no. 5, p. 719-722, Sept. 2005

18. NOLLA, C. M. The development of the permanent teeth. ASDC J. Dent. Child., Chicago, v. 27, no. 4, p. 254-266, 1960.

19. RICHARDSON, M. E. The development of third molar impaction and its prevention. Int. J. Oral Surg., Copenhagen, v. 10, p. 122-130, 1981. Supplement 1.
20. RICHARDSON, M. E. Lower molar crowding in the early permanent dentition. Angle Orthod., Appleton, v. 55, no. 1, p. 51-57, Jan. 1985

21. RICHARDSON, M. E. Lower third molar space. Angle Orthod., Appleton, v. 57, no. 2, p. 155-161, Apr. 1987.

22. RICHARDSON, M. E. The effect of mandibular first premolar extraction on third molar space. Angle Orthod., Appleton, v. 59 , no. 4, p. 291-294, Winter 1989

23. RICHARDSON, M. E. The role of the third molar in the cause of late lower arch crowding: a review. Am. J. Orthod. Dentofacial Orthop. St. Louis, v. 95, no. 1, p. 79-83, Jan. 1989.

24. RICHARDSON, M. E. O terceiro molar: uma perspectiva ortodôntica. Rev. Dental Press Ortodon. Ortop. Facial, Maringá, v. 3, n. 3, p. 103-117, maio/jun. 1998.

25. RICHARDSON, M. E.; DENT, M. Some aspects of lower third molar eruption. Angle Orthod., Appleton, v. 44, no. 2, p. 141-145, Apr. 1974.

26. SCHULHOF, R. J. Third molars and orthodontic diagnosis. J. Clin. Orthod., St. Louis, v. 10, no. 4, p. 272-281, Apr. 1976.

27. SILLING, G. Development and eruption of the mandibular third molar and its response to orthodontic therapy. Angle Orthod., Appleton, v. 43, no. 3, p. 271-278, July 1973.

28. SLODOV, J.; BEHRENTS, R. G.; DOBROWSKI, D. P. Clinical experience with third molar Orthodontics. Am. J. Orthod. Dentofacial Orthop., St. Louis, v. 96, no. 6, p. 453-461, Dec. 1989.

29. STAGGERS, J. A.; GERMANE, N.; FORTSON, W. M. A comparison of the effects of first premolar extractions on third molar angulation. Angle Orthod., Appleton, v. 62, no. 2, p. 135-138, Summer 1992.

30. TAVANO, O.; URSI, W. J. S.; ALMEIDA, R. R.; HENRIQUES, J. F. C. Determinação de linhas de referência para medições angulares em radiografias ortopantomográficas. Odontol. Mod., Rio de Janeiro, v. 16, n. 9, p. 22-25, set. 1989.

31. URSI, W. J. S.; ALMEIDA, R. R.; TAVANO, O.; HENRIQUES, J. F. C. Assessment of mesiodistal axial inclination through panoramic radiography. J. Clin. Orthod., St. Louis, v. 24, no. 3 , p. 166-173, Mar. 1990.

\section{Endereço para correspondência}

Osmar Aparecido Cuoghi

Rua José Bonifácio, 1193 - Faculdade de Odontologia de Araçatuba CEP: 16.015-050 - Araçatuba/SP

E-mail: osmar@foa.unesp.br 